\title{
Analysis and Characterisation of Heart Rate Variability (HRV) Data of Different Sets of Subjects Using Nonlinear Measure (Approximate Entropy)
}

\author{
CH.RenuMadhavi and A.G.Ananth
}

\begin{abstract}
The human heart is a nonlinear system because the heart rhythm is modulated by the Autonomic nervous system (ANS).The modulation of the heart rate about its mean value is called Heart rate variability (HRV). Nonlinear analysis of HRV is helpful to assess the cardiac health noninvasivly.. The analysis of HRV data of different sets of subjects is done using ApEn.The autonomic dysfunction also causes cardiac malfunction. The Subjects involved in meditation is taken as one set. The other set is depressed subjects. The third set is congestive heart failur(CHF) subjects. The fourth set is healthy subjects. Also The effect of ANS on cardiac functioning is investigated, before and after the meditation for the set of meditation subject and also for the set of depressed subjects. The cardiac health status is also investigated for the set of Congestive heart failure (CHF) subjects and and healthy subjects. It has been found that the ApEn values indicated improved cardiac health after meditation.The ApEn values of depressed subjects is lower than that of healthy subjects. The high values of ApEn for healthy subjects suggest that the functioning of heart is dynamic. The lower ApEn values of the depressed subjects observed indicate the presence of the Autonomic dysfunction, which in turn effected cardiac function indirectly causing heart to exhibit less dynamism.. The CHF data showed the Apen values lower than healthy subjects indicating cardiac ill health.
\end{abstract}

Index Terms-Heartratevariability,,Nonlinear,Approximate entropy,meditation,depressed

\section{INTRODUCTION}

It is known that Electrocardiogram (ECG) represents the electrical activity of the heart. This electrical activity is generated by SinoAtrial (SA) node which is a biological pacemaker present in the heart. The SA node is controlled by ANS which modulates the normal heart rhythm through sympathetic nervous system (SNS )and parasympathetic nervous system(PNS).So the heart is not a linear system, can be effected by nonlinear contributions. Therefore, it is considered as a complex system.

Heart rate is the reciprocal of the time interval between two consecutive $\mathrm{R}$ peaks of ECG. Typical value of heart rate is 72 beats per minute. The variation of Heart rate from its mean

CH.RenuMadhavi is with the Dept, of Instrumentation,R.V.College of Engineering, Bangalore, India(renumadhavi@yahoo.comm).

A.G.Ananth is with the Dept of Telecommunication, R.V. College of Engineering, Bangalore, India (antisro@yahoo.com). value is called Heart Rate Variability (HRV). The HRV can shows the presence of disease and gives indications about the threatening diseases. Hence for the study of the Cardio-autonomic abnormalities HRV has become an important noninvasive tool. HRV analysis is helpful to estimate the cardiac health and the state of autonomic nervous system which is responsible for affecting the cardiac functionality. [1].

HRV analysis is done using Time domain methods,Frequency domain methods, nonlinear methods and Geometrical methods. The time domain methods are simple, but can not distinguish between the SNS and PSN components of HRV. Frequency domain methods estimate the power spectral densities by using both parametric and nonparametric methods. The nonparametric method of analysis is done by applying Fast Fourier Transforms (FFT) or periodograms The frequency domain can show the cause producing the effect in terms of the frequency components and power spectral density.. These methods have a limitation of spectral leakage, which will hide the weaksignal present in the data., this limitation is over come by using parametric power spectral estimation methods namely Autoregressive (AR) modeling [ 1].

The linear methods of analysis apply timeseries analysis, where the parameters such as mean of the data,standard deviation, variance are estimated.

Spectral analysis is helpful to see the the sympathetic component in low frequency range, which is from $0.04 \mathrm{~Hz}$ $0.15 \mathrm{~Hz}$. The parasym apathetic component can be seen in highfrequency, whose range is $0.15 \mathrm{~Hz}-0.4 \mathrm{~Hz}$.

The geometrical methods of analysis is done by using Poincare plots and recurrence plots. To draw Pioncare plots it is required to take all the RRi interval data on Xaxis and $R R i+1$ interval data on $Y$ axis. The graph is plotted for RRi Verses $R R i+1$.The shape of the graph thus obtained is used to find whether the subject is normal or abnormal.

Recurrence plots also are used for the graphical representation about the status of the heart.Generally the patterns of graphs obtained will be different for different datasLooking at the Recurrence plot also the health of the heart can be predicted as whether it is normal or abnormal..

The processes generated by biological systems aredescribed more effectively by various Nonlinear measures.[1]such as correlation dimension(CD), Detrendedfluctuation analysis (DFA).Approximate Entropy 
(ApEn), Sample Entropy (SampEn), Largest'Lyapunov Exponent (LLE), Fractal dimension (FD), Hurst exponent (H).The geometrical methods applied for the visualization of HRV as a graph are Poincareplots and Recurrence plots., The pattern of the plots indicates the cardio autonomic state.[1] ApEn is the measure of complexity of the time series data If ApEn is larger the system is complex, if its value is smaller the system is said to be regular. [2, 3, 4].

Application of nonlinear analysis to ECG started two decades ago. .Extensive work has been carried out nonlinear analysis of HRV. The nonlinear nature of physiological signals such as ECG suggests the application of nonlinear techniques for the diagnostic and classification of the biological system [1]. ApEn can be used as parameter to measure the complexity of a biological system $[2,3,4]$.

Nonlinear techniques were applied for the classification of various types of cardiac abnormalities $[1,10,11,12]$ it was suggested that linear and nonlinear methods are applicable to various cases with HRV such as blood pressure, myocardial infraction, cardiac arrhythmias, diabetes, renal failure , gender and age, drugs , smoking,sleep in infants[1,10,11,12]

Recent results have suggested that the fluctuations in time between beats in a healthy heart may be due to an intrinsic variability and the nonlinear nature of the autonomic nervous system control exhibiting a complex behavior. ApEn, SampEn,LLE,D2, Standard deviation1 (SD1 )Standard deviation 2(SD2) were used as nonlinear measures for $\mathrm{PVC}, \mathrm{VF}, \mathrm{AF}$ subjects along with healthy subjects and further classified the data.[15]

The non linear analysis of HRV has significant role in the assessment of cardiac health status. In this work the effect of nonlinear entropy measure ApEn of HRV on healthy and different disease subjects is investigated .Further the effect of meditation on HRV is studied.

\section{DAta AnAlysis And Methodology}

For the present analysis the ECG signals were obtained from diferent sources. The sources of ECG data acquisition are the Powerlab I nstrument and the meditation data derived from Physionet[18].

ECG signals of pre and post meditation were acquired from seven healthy subjects using the PowerLab instrument. The data is collected from lead 2 at a sampling frequency of $500 \mathrm{~Hz}$. The RR intervals were separated using the inbuilt filters in the instrument and then applied as input data to the program for estimation of ApEn.

The sample of the ECG intervals got from the Power Lab systems is as shown in figure 1 below.

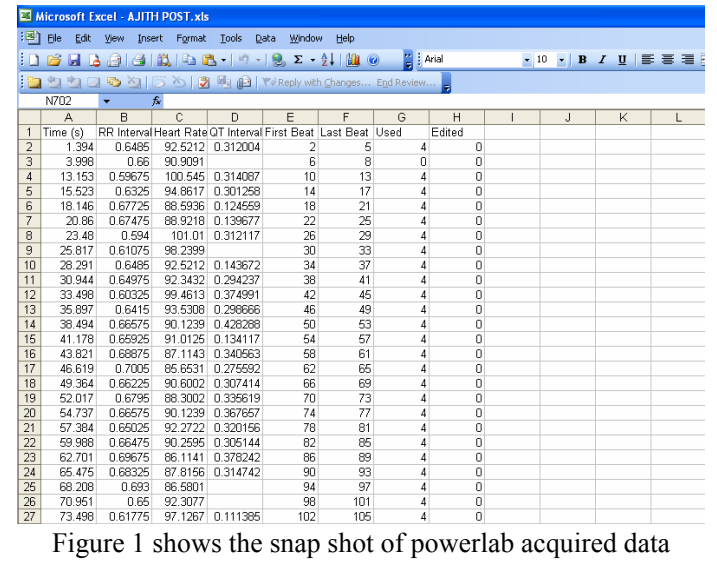

ECG signals of three depressed subjects and three healthy subjects were acquired using the PowerLab instrument

The five different types of data are used for the present analysis. The first type is the pre and post meditation of seven healthy subjects. The second type of data is the data of three healthy subjects derived from powerlab.

The third type of data is the data of three depressed subjects derived from powerlab. The fourth type of data is the congestive heart failure(CHF) data derived from Physionet[18], and the fifth type is the meditation data derived from Physionet[17].

Four sets RR intervals of pre and post meditation were acquired from Physiobank [17]. The nonlinear complexity measure is done by implementing the algorithm for ApEn proposed by Pincus $[2,3,4]$ using MATLAB

The nonlinear measure estimated for the data acquired is Approximate Entropy(ApEn). The heart rate dynamics can be quantified using number of nonlinear measures one of them is ApEn.The ApEn can be applied to short length of data hence it is selected.

Apen is a nonlinear measure that signifies the complexity of the applied time series data.The calculation of ApEn requires three parameters namely $\mathrm{N}$, length of the data, $\mathrm{r}$ the threshold and $\mathrm{m}$ the embedding dimension. The value of $\mathrm{m}$ can be 1 or 2 and $r$ can be 0.15-0.2.

The values of ApEn determines whether the data is random or deterministic.Lower the value of ApEn the data is deterministic and higher the value of ApEn the data is random.

ApEn is calculated by first finding the frequency with which all the ' $\mathrm{m}$ ' point patterns in the sequence are close to each other,,let this frequency be ' $\mathrm{kl}$ ', and finding the frequency with which all the ' $m+1$ ' point patterns in the sequence are close to each other,,let it be ' $\mathrm{k} 2$ ', then fiding the difference between $\mathrm{k} 1$, and $\mathrm{k} 2$ gives the value of ApEn..The value of $k 1$ is estimated by calculating the natural logarithm of correlation integral of $\mathrm{m}$ point patterns in the sequence, for $\mathrm{i}=1$ to $\mathrm{N}-\mathrm{m}+1$, take the average of the correlation integrals. To compute $\mathrm{k} 2$ the same procedure as done for $\mathrm{k} 1$ is repeated but now $\mathrm{m}+1$ point patterns need to be considered for the calculation of correlation integral..The ApEn is estimated as the the difference between $\mathrm{k} 1$ and $\mathrm{k} 2$. 


\section{RESUlt AND DisCUSSION}

The ApEn values of subject before and after meditation are computed for the data acquired from Powerlab has been shown in figure2.The figure shows that the ApEn values of pre meditation subjects indicates a higher value and increased to little more higher value for post meditation subjects.

It is evident from the figure that the ApEn values determined for post- meditation subjects increased when compared to premeditation subjects. The values of ApEn computed for data obtained using powerlab are presentedin table1.

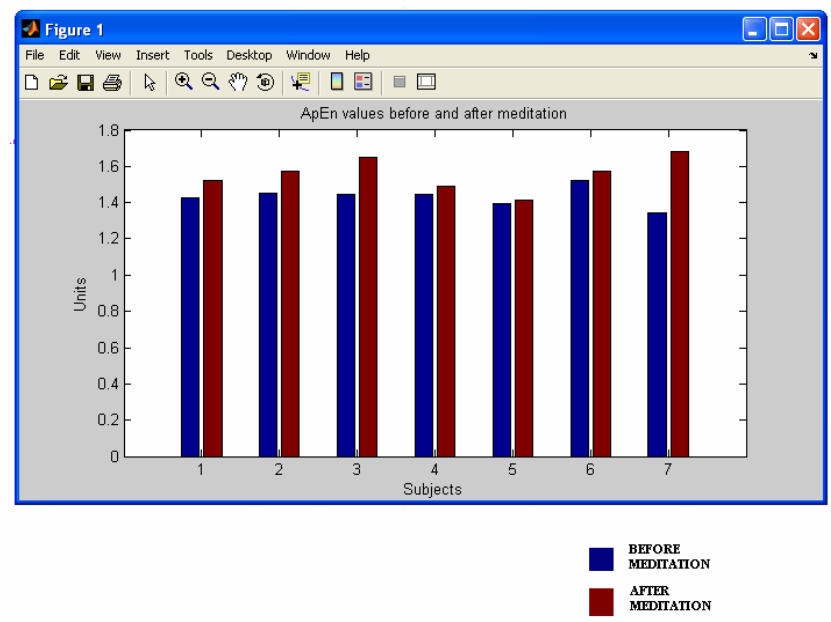

Figure2: shows ApEn values before and after meditation of powerlab data

The mean ApEn values are computed for healthy subjects before and after meditation as shown in figure $2 \mathrm{a}$

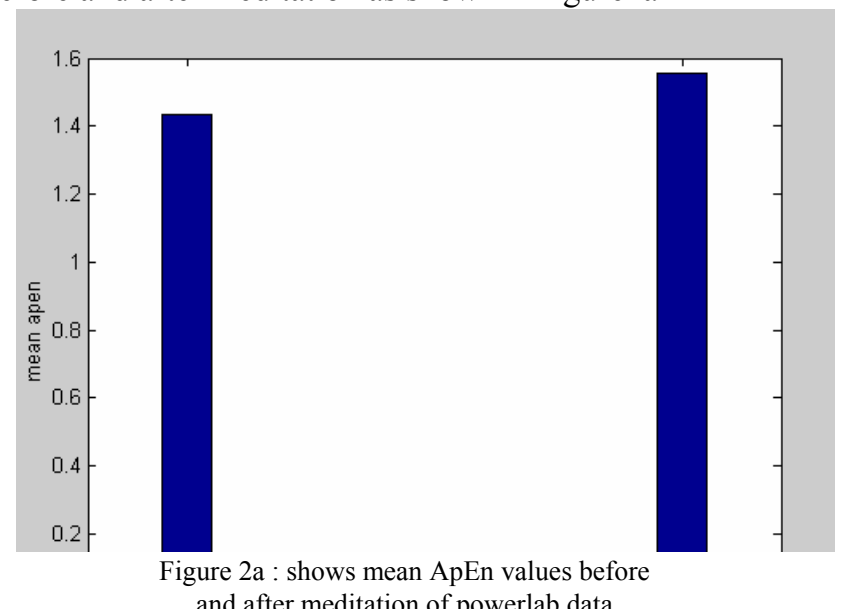

The figure shows a high mean value for premeditation data and an increase in its value after meditation. Showing the evidence of improved health

TABLE 1 Computed Values of APen of Powerlab Data

\begin{tabular}{|l|l|l|}
\hline Sample & ApEn Premeditation & ApEn Post meditation \\
\hline 1 & 1.425804 & 1.521591 \\
\hline 2 & 1.454506 & 1.570543 \\
\hline 3 & 1.447770 & 1.648860 \\
\hline 4 & 1.447763 & 1.492151 \\
\hline 5 & 1.396951 & 1.415407 \\
\hline 6 & 1.521463 & 1.571821 \\
\hline 7 & 1.343834 & 1.684213 \\
\hline Mean & $\mathbf{1 . 4 3 4 0}$ & $\mathbf{1 . 5 5 7 8}$ \\
\hline
\end{tabular}

These results can be used to monitor the variation in heart dynamics. For premeditation healthy subjects ApEn value is high and the functioning of the heart is very dynamic and HRV is non regular. For the post meditation healthy subjects the ApEn values increased still higher indicating that the dynamism of the heart is more

The ApEn values of subjects whose data is acquired from Physionet [17] are computed after performing the physical activity (meditation). The ApEn values are computed for pre and post meditation cases and the results are tabulated in table 2 shown below, and indicated in the figure 3.

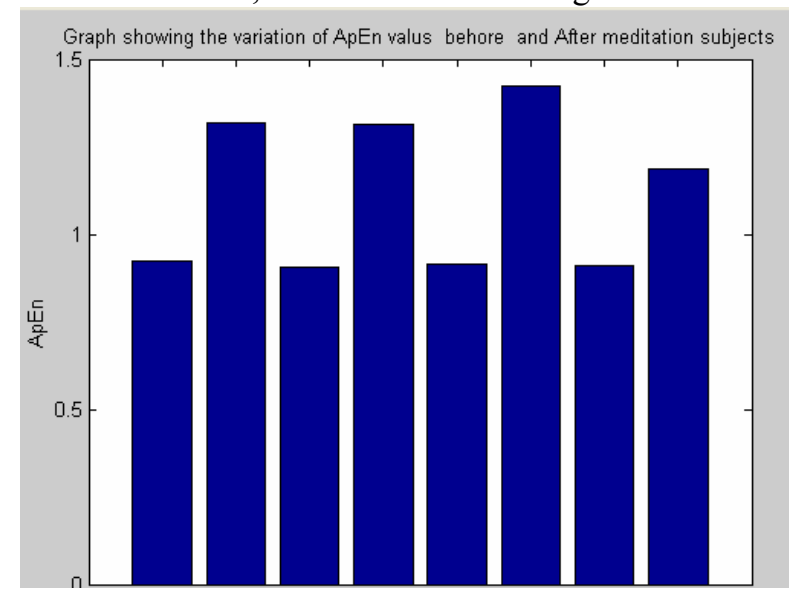

Figure3: shows ApEn values before and after meditation of physionet data

It is evident from the figure that the ApEn valuesdetermined for premeditation are low and for post meditation subjects the values increased.

The mean ApEn values computed for pre and post meditation for physionet data is shown below in figure $3 a$

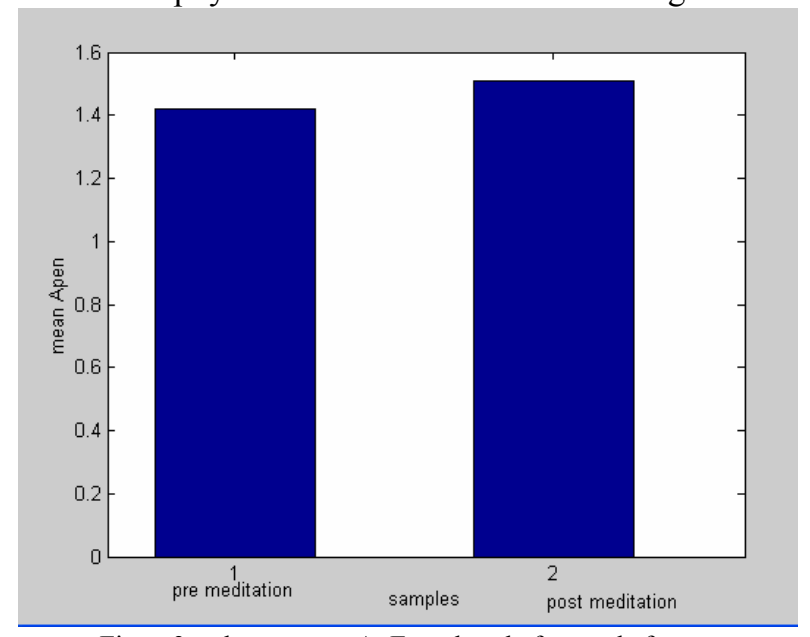

Figure3a: shows mean ApEn values before and after meditation of physionet data

From figure it is clearly seen that the mean ApEn value is less before meditation and increased after meditation indicating an improvementThe lower value of ApEn before meditation suggests that the subjects heart is less dynamic,and the increased value ofApEn after meditationindicates improved heart dynamism. 
TABLE 2 COMPUTED VAlues of APEN OF PHysionet DAta[1]

\begin{tabular}{|c|c|l|}
\hline sample & $\begin{array}{l}\text { ApEn } \\
\text { Premeditation }\end{array}$ & $\begin{array}{l}\text { ApEn } \\
\text { Post } \\
\text { meditation }\end{array}$ \\
\hline PH1 & 0.9215 & 1.3178 \\
\hline PH2 & 0.90466 & 1.3153 \\
\hline PH3 & 0.91423 & 1.4230 \\
\hline PH4 & 0.9116 & 1.1882 \\
\hline mean & 0.9129 & 1.311 \\
\hline
\end{tabular}

It can be observed from the table 2 that the Approximate entropy value increased after meditation. The mean value of post meditation is more than the mean of premeditation.

The values of ApEn for premeditation is lower compared to postmeditation in this case.

In order to emphasize the significance of the results the ApEn values for both healthy and depressed subjects is computed. As shown in figure4 It is observed from the figure that the ApEn value is significantly high for healthy subjects as compared to depressed subjects. This result signifies the role played by ANS in controlling HRV and indicating its indirect effect. on heart

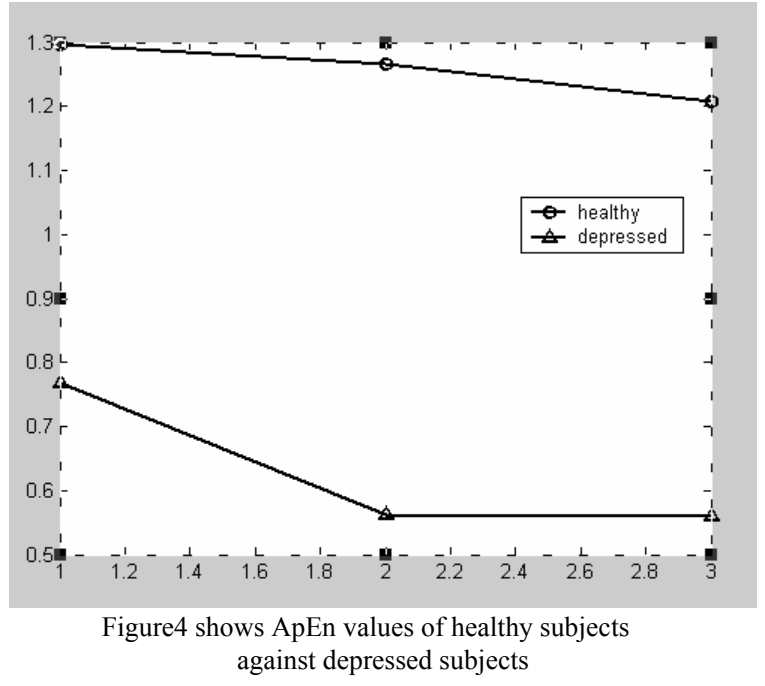

Further it can be seen from the figure 5 that ApEn values are higher for healthy subjects and lower ApEn for Congestive heart failure(CHF) subjects..

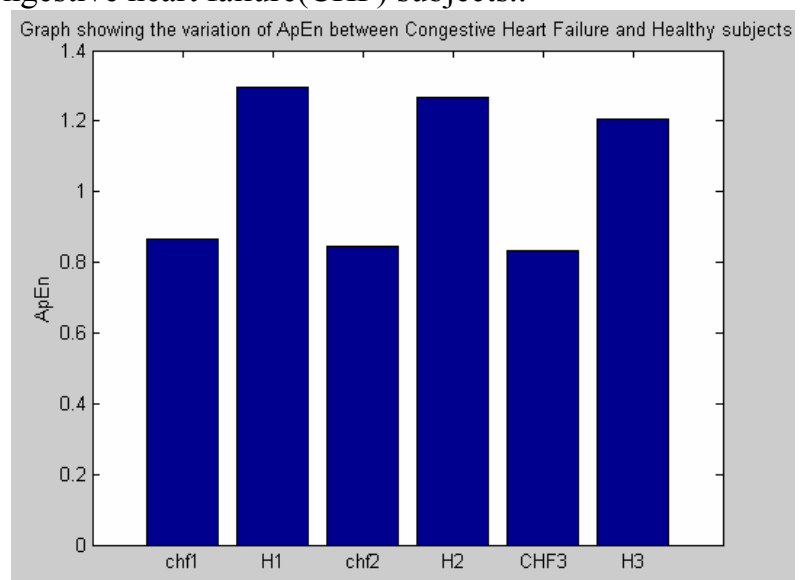

Figure5 shows Apen values of achf subjects and healthy subjects [18]
It is evident from figure that these results can be used to monitor healthy or unhealthy status of a heart. Furthe it is bserved that ApEn value is high and the functioning of the heart is very dynamic for healthy subjects, and less dynamic for the CHF subjects.

The mean ApEn value of CHF subjects is found to be lesscompared to healthy subject's mean ApEn value.

The mean ApEn values are estimated for healthy subjects,depressed subjects, healthy subjects involved in physical activity, Subjects whose health sate is unknown but involved in physical activity and CHF subjects is shown in figure6.

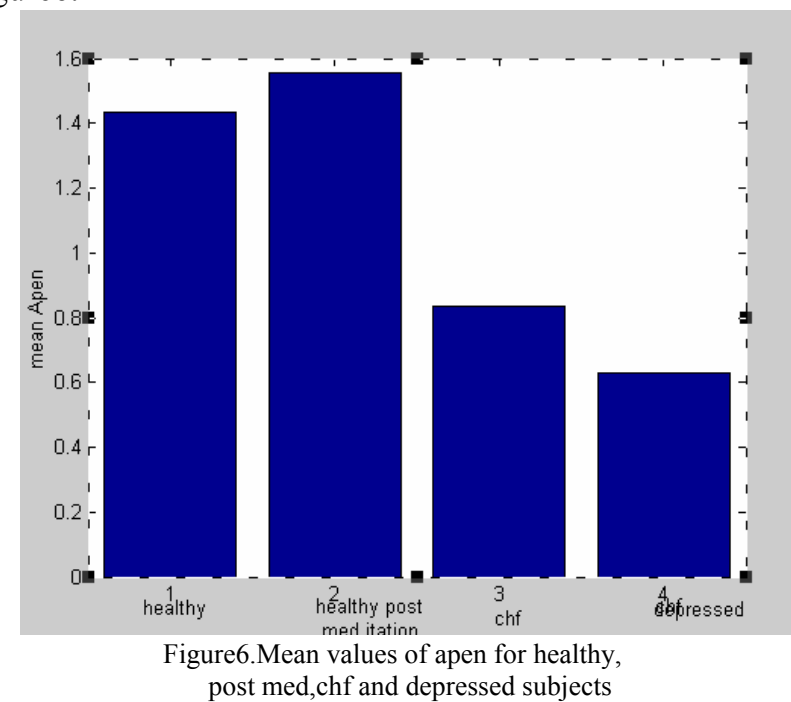

It is clearly seen from the figure that the mean ApEn value of a healthy subjects is high and the value increased still high after meditation. Also it is evident from the figure that the mean ApEn value of CHF subject is low and it further reduced to a lower value for depressed subjects.

\section{CONCLUSIONS}

(1) The analysis of HRV data of healthy subjects suggested a high value for ApEN Indicating the complexity of HRV thus shows that heart is healthy agreeing with the earlier works

(2) ApEn values computed for the known subjects who are healthy indicated high ApEn values before meditation and increased little higher after meditation. This observation suggests that the functioning of the heart is improved further after meditation

(3) The ApEn values computed for the meditation data obtained from Physionet,where the details of the subjects is unknown.The results indicated a Lower ApEn value for pre meditation and increased o higher values after meditation. The increased values of ApEn after meditation indicates improved heart health. The initial low value indicating the less dynamism in the heart activity may be the subjects are not healthy.

(4) The ApEn values of depressed subjects exhibited a lower value compared to the healthy.This lower value indicates the indirect effect of ANS dysfunction effecting the cardiac function .

(5) It is evident from the results that the ApEn values of 
CHF subjects are lower compared to healthy subjects agreeing with the earlier works

The analysis of the HRV data in using ApEn as a nonlinear measure is helpful to identify noninvasively health status (normal/abnormal)of the subjects, further it is observed that the health status improved due to meditation for a healthy subject and also for unhealthy subjects.The ApEn values of depressed subjects attributed to the ANS dysfunction. The meanApen value of the depressed subjects is the lowest of all values calculated.

\section{ACKNOWLEDGEMENTS}

We acknowledge the encouragement and support given by the Prof.K.V..Padmaja, HOD, Department of Instrumentation and Prof. K.N.RrajaRao, Principal , R.V. College of Engineering, Bangalore for their constant encouragement and support for carrying out the present research work.We would also like to thank Dr.Jayasri and Dr.Vinay for providing us with the required data for this work.

\section{REFERENCES}

[1] U.Rajendra Acharya et.al "Heart rate Variability :a review"Med.Bio.Eng. Compu t(2006)44:1031-1051

[2] Pincus SM "Approximate entropy as a measure of system complexity" Proc.Natl.Acad.Sci.USA 88:2297-2301,1991

[3] S.M.Pincus et.al "a regularity statistic for medicl data analysis"J.Clin.Monitor,7:335-345,1991

[4] S.M.Pincus et.al "Greater signal regularity may indicateincreasedsystemsolation”Math.Biosci122:161-181,1992 regularity
[5] J. S. Richman and J. R. Moorman"Physiological time-series analysis using approximate entropy and sample entropy Am. J. Physiol. HeartCirc. Physiol., vol. 278, pp. H2039-2049, Jun. 2000.

[6] Gley Kheder et.al"Application of a nonlinear dynamic method in the analysis of the HRV (HeartRate Variability) towards clinical application: Tiresome diagnosis", 0-7803-9521-2/06/\$20.00 \$2006 IEEE

[7] G Krstacic1,.et.al The Chaos Theory nd Non-linear Dynamics in Heart Rate Variability in Patients with Heart Failure Computers in Cardiology 2008; 35:957-959

[8] Roberto Hornero .et.al ", Interpretation of Approximate Entropy: nalysis ofIntracranial Pressure Approximate Entropy DuringAcute Intracranial Hypertension"IEEE transactions on biomedical engineering, vol. 52, no. 10, october 20051671

[9] Xiaobo Miao.et.al" Heart rate variability characterization usingCorrelation Dimension"0-7803-7523-8/02/\$17.00c2002IEEE

[10] U. Rajendra Acharyaa, et.al"Classifi_cation of heart rate data using artifi_cial neural networkand fuzzy equivalence relation" Pattern Recognition 36 (2003) $61-68$

[11] RajendraU.Acharya.et.al"Compehensive analysis of cardiachealthusing heart rate signals"Physiol.Meas.J.25:1130-1151,2004

[12] Rajendra Acharya U.et.al" Heart rate analysis in normal subjectsofvarious age groups"BioMedical Engineering OnLine20 July 2004

[13] Y.Sun.et.al.'life threatening ventricular arrhythmia recognition bynonlinear descriptor" biomedical engineering online, 2004

[14] Z.iafu zhu.et.al "Comparitive analysis of heart rate variability between morbid group based on Correlation Dimension"978-4244-1748-3/08/\$25 2008 ,IEEEE PP 2252-2255

[15] Sohrab Behnia et.al, "On the Calculation of Chaotic Features for Nonlinear Time Series" Chinese Journal of Physics Vol. 46 , no. 4 August 2008

[16] Rajendra Acharya U.et.al"Cardiac Health Diagnosis using WaveletTransformation and Phase Space Plots"Proceedings of the 2005 IEEEEngineering in Medicine and Biology 27th Annual ConferenceShanghai, China, September 1-4,

[17] www.physionet.org/physiobank/meditation data

[18] www.physionet.org/physiobank/mitdatabase 